further opportunities to help them maintain the world lead they gained after UK-5, an X-ray satellite launched in 1975. They feel that the data from EXOSAT, ESA's large X-ray observatory, will be spread on the ground too thinly to be of much use to them. "The future of British astrophysics does not lie with ESA" commented one British high energy astronomer.

Recently the SRC has been devoting some thought to ways of providing UK scientists with more space opportunities and of cutting the cost of space science. It is considering collaborating with NASA on a multidisciplinary refurbishable satellite (MRS) to be launched and refurbished by NASA's Space Shuttle. The idea is to increase the lifetime and usefulness of a single satellite by repairing it and altering its payload either in orbit or on the ground. The SRC hopes that an MRS could be a very cheap way of doing both astrophysics and geophysics experiments. There are some fears, however, especially among geophysicists that by trying to please everyone it will please no one.

The SRC would like to involve ESA in studies on the MRS, but enthusiam for the idea within ESA is low. There are doubts about its feasibility and worries that it would mean yet another collaboration with NASA. Nevertheless, ESA will at least be obliged to look into the idea although it could not be studied in time for ESA's next project decision at the end of 1979.

West Germany's policy is to conduct as much of its space science through ESA as possible and it would actually like to increase its contribution to ESA's mandatory science budget. However, it could only do this if all member states agreed to increase their contributions accordingly. In the long term, Germany would like to see funds diverted to science from applications. "Applications should only go on in ESA for a short time," says Dr H. Strub of the BMFT, "then they must be marketed and taken out of ESA's control. In science ESA runs and develops satellites but in applications, the users must take this over once the technology has got off the ground". In particular, Germany would like to see more spent on science when ESA's own launcher, Ariane, and Spacelab near the end of the most expensive phases of their development. By the mid-1980s, it would like ESA's entire budget to level off at 350 mau (about $£ 230 \mathrm{~m}$ ) at today's prices (it is now about 530 mau, £345m) and most of it to be spent on science and new fields to ESA bordering on applications and science such as remote sensing and climatology.

The greatest pressure on ESA to do planetary science comes from
Germany. German scientists would like to consolidate the experience they gained from the Helios probes, two solar physics missions launched in 1974 and 1976 in collaboration with NASA. The French would also like to build on their collaborations with the Soviet Union on a number of planetary missions. Germany is also considering collaborating with NASA on Robi, a cheap X-ray satellite.

Scientists within the SAC feel many of ESA's problems could be solved if member states agreed to increase the mandatory science budget and ESA could increase its cost-effectiveness. Professor Klaus Pinkau of the Max Planck Institute fur Extraterrestiche Physik recently sent out a questionnaire to European space scientists canvassing opinion on ESA. "The science budget doesn't need a six-fold increase" Professor Pinkau told Nature. "This would be out of proportion to the funding of the rest of science". He would like to see the mandatory science budget restored to its pre-1971 level, about 100 mau $(£ 65 \mathrm{~m})$ at today's prices, and would like ESA to make efforts to increase its cost-effectiveness by at least $20 \%$. Over a ten year period, he says, ESA

\section{The multi-discipline refurbishable satellite}

THE MRS is one country's (the UK's) attempt to find more flying time outside the auspices of ESA. Under consideration by the Astronomy, Space and Radio Board of the Science Research Council, it would use The Godard Space Flight Center's kit-form Multimission Modular Spacecraft (MMS) as a base (see figure) and erect on it instruments to serve both astronomers and geophysicists. A study is underway (it will report in January) at British Aerospace, Stevenage, to assess the difficulties of combining the instruments on the same vehicle. "And it doesn't make life easy" said one scientist.

The MRS would be a collaboration with NASA, which is interested in flying high energy astronomy experiments. But NASA is not concerned with flying geophysics on this mission; so geophysicists in the UK are suspicious that the exercise is one designed by high energy astronomers to 'sell' the project to the Science Research Council, which is conscious of the need to serve as many scientists as possible.

Funds would be needed by about the end of 1980, for a launch from the Space Shuttle in 1985 . The UK would assemble the satellite, at a cost of $£ 15-£ 20$ m-twice the cost of UK6, for example. But the MRS could take a payload of up to $1,000 \mathrm{~kg}$, and could should be able to fly one mission a year if it built five missions costing 50 mau each, four costing 120 mau and one very good and expensive one costing 250 mau.

To achieve this, however, ESA would have to make special efforts to keep the cost of small satellites within the 50 mau limit. Professor Pinkau suggests that facilities on the ground could be used more efficiently so that the science budget does not have to pay for facilities which are not being used for some part of the year. "Good management should show where you lose money", he says. The industrial policy might also have to be revised so that the principle of fair return would apply over say ten years.

This scheme, he feels could incorporate materials and life sciences into the mandatory budget. Earth resources would have to remain outside the budget but could still be refereed within the procedure of the SAC. Such a scheme however is dependent on the member states agreeing to increase the science budget and on ESA achieving a substantial reduction in costs. The former lies wthin the power of national governments. The latter is up to ESA.

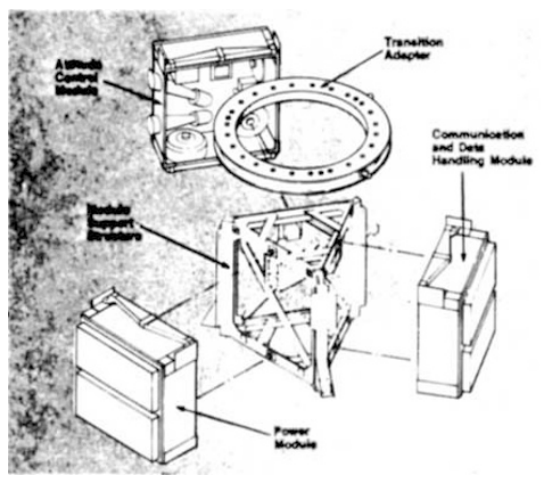

Exploded view of MMS basic modules and elements

have a continuous data rate an order of magnitude greater than UK6.

Atmospheric physics may be the casualty of the multidiscipline confrontation, as its requirements are least compatible with astronomy. But the MRS is retrievable and refurbishable and is designed to make use of the supposed reduced costs of reuse of the MMS base and some instrumentation. Geophysics could get a better look in on a subsequent launch.

But everything depends on whether an agreemennt can be reached with NASA on the first and subsequent flights-as always with such collaborations-and, ultimately, on the uncertain cost of operations with the shuttle. 